\title{
Boundedness of higher order commutators of oscillatory singular integrals with rough kernels
}

\author{
by
}

\author{
HuOXIONG Wu (Xiamen)
}

\begin{abstract}
The author studies the commutators generated by a suitable function $a(x)$ on $\mathbb{R}^{n}$ and the oscillatory singular integral with rough kernel $\Omega(x)|x|^{n}$ and polynomial phase, where $\Omega$ is homogeneous of degree zero on $\mathbb{R}^{n}$, and $a(x)$ is a BMO function or a Lipschitz function. Some mapping properties of these higher order commutators on $L^{p}\left(\mathbb{R}^{n}\right)$, which are essential improvements of some well known results, are given.
\end{abstract}

1. Introduction. We will work on $\mathbb{R}^{n}, n \geq 2$. Let $\Omega$ be a function homogeneous of degree zero with mean value zero on the unit sphere $S^{n-1}$. Define the oscillatory singular integral operator $T$ by

$$
T f(x)=\text { p.v. } \int_{\mathbb{R}^{n}} e^{i P(x, y)} \frac{\Omega(x-y)}{|x-y|^{n}} f(y) d y,
$$

where $P(x, y)$ is a real-valued polynomial on $\mathbb{R}^{n} \times \mathbb{R}^{n}$. Let $k$ be a positive integer and $a(x)$ a suitable function on $\mathbb{R}^{n}$. Define the $k$ th order commutator $T_{a, k}$ generated by $T$ and $a$ by

(2) $T_{a, k} f(x)=$ p.v. $\int_{\mathbb{R}^{n}} e^{i P(x, y)}(a(x)-a(y))^{k} \frac{\Omega(x-y)}{|x-y|^{n}} f(y) d y, f \in C_{0}^{\infty}\left(\mathbb{R}^{n}\right)$.

The operators (1) and (2) are called the oscillatory operators with polynomial phase. As is well known, operators of this type are very useful in the study of Hilbert transforms along curves, singular integrals supported on lower dimensional varieties, singular Radon transforms etc. There has been a considerable amount of relevant research since Ricci and Stein's pioneering paper [20] (see e.g. [3, 15, 10, 7, 13]). In this paper, we will focus on the operator (2) in two cases described below.

1.1. Commutators generated by a BMO function. For $a \in \mathrm{BMO}\left(\mathbb{R}_{+}\right)$ (the radial BMO function class), Ding and Lu [7] (resp., Lu and Wu [13])

2000 Mathematics Subject Classification: 42B20, 42B25.

Key words and phrases: oscillatory singular integral, commutator, BMO, BLO, block, Lipschitz spaces, rough kernel.

Supported by the NSF of China, Grant 10271016. 
proved the weighted $L^{p}$-boundedness of $T_{a, k}(1<p<\infty)$ if $\Omega \in L \log ^{+} L\left(S^{n-1}\right)$ (resp., $\Omega \in B_{q}^{0,0}\left(S^{n-1}\right)$, where $B_{q}^{0,0}$ denotes the block space introduced by Jiang and $\mathrm{Lu}[12])$. For general $a \in \operatorname{BMO}\left(\mathbb{R}^{n}\right)$, Ding [5] showed that $T_{a, k}$ is bounded on $L^{p}\left(\mathbb{R}^{n}\right)(1<p<\infty)$ with bound $C\|a\|_{\mathrm{BMO}\left(\mathbb{R}^{n}\right)}^{k}$ independent of the coefficients of $P(x, y)$ (also see [9]) if $\Omega \in \bigcup_{r>1} L^{r}\left(S^{n-1}\right)$. Recently, Ma and $\mathrm{Hu}$ [16] extended the result in [5] to the case of $\Omega \in L\left(\log ^{+} L\right)^{k+1}\left(S^{n-1}\right)$ for $p=2$.

Obviously, the condition that $\Omega \in L\left(\log ^{+} L\right)^{k+1}\left(S^{n-1}\right)$ greatly depends on the order $k$ of $T_{a, k}$. Moreover, for $p \neq 2,1<p<\infty$, Ma and Hu [16] did not obtain the corresponding result. It is natural to ask whether there exists a condition on $\Omega$, which is strictly weaker than $\Omega \in \bigcup_{r>1} L^{r}\left(S^{n-1}\right)$ and independent of $k$, such that $T_{a, k}$ is bounded on $L^{p}\left(\mathbb{R}^{n}\right)$ for $1<p<\infty$. In this paper, we will give a positive answer to the above problem. Before stating the main result, we introduce some concepts (see [12]).

Definition 1. A $q$-block on $S^{n-1}$ is an $L^{q} \quad(1<q \leq \infty)$ function $b(\cdot)$ that satisfies

$$
\text { (i) } \operatorname{supp}(b) \subseteq Q, \quad \text { (ii) }\|b\|_{L^{q}\left(S^{n-1}\right)} \leq|Q|^{1 / q-1},
$$

where $Q=S^{n-1} \cap\left\{y \in \mathbb{R}^{n}:|y-\varsigma|<\varrho\right.$ for some $\varsigma \in S^{n-1}$ and $\left.\varrho \in(0,1]\right\}$.

Definition 2. The block spaces $B_{q}^{0,0}$ on $S^{n-1}$ are defined by

$$
B_{q}^{0,0}\left(S^{n-1}\right)=\left\{\Omega \in L^{1}\left(S^{n-1}\right): \Omega\left(y^{\prime}\right)=\sum_{s} C_{s} b_{s}\left(y^{\prime}\right), M_{q}^{0,0}\left(\left\{C_{s}\right\}\right)<\infty\right\},
$$

where each $C_{s}$ is a complex number, each $b_{s}$ is a $q$-block supported in $Q_{s}$, and

$$
M_{q}^{0,0}\left(\left\{C_{s}\right\}\right)=\sum_{s}\left|C_{s}\right|\left\{1+\log ^{+} \frac{1}{\left|Q_{s}\right|}\right\} .
$$

It should be pointed out that the method of block decomposition for functions was invented by Taibleson and Weiss [21] in the study of convergence of Fourier series. Later on, many applications of the block decomposition to harmonic analysis were discovered (see e.g. $[1,11,12,13,14])$. For further background and information about spaces generated by blocks and their applications to harmonic analysis, one can consult the book [12]. In particular, Keitoku and Sato [11] showed that for any $q>1$,

$$
\bigcup_{r>1} L^{r}\left(S^{n-1}\right) \subset B_{q}^{0,0}\left(S^{n-1}\right),
$$

which is a proper inclusion. And we easily see from [11] that $B_{q}^{0,0}\left(S^{n-1}\right)$ is not contained in $L\left(\log ^{+} L\right)^{1+\varepsilon}\left(S^{n-1}\right)$ for any $\varepsilon>0$ although the relationship between $B_{q}^{0,0}\left(S^{n-1}\right)$ and $L \log ^{+} L\left(S^{n-1}\right)$ remains open. 
Definition 3 (see [4]). A locally integrable function $a(x)$ will be said to belong to $\mathrm{BLO}\left(\mathbb{R}^{n}\right)$ if there is a constant $C$ such that for any cube $Q$,

$$
m_{Q}(a)-\inf _{x \in Q} a(x) \leq C,
$$

where $m_{Q}(a)=|Q|^{-1} \int_{Q} a(x) d x$.

If $a \in \mathrm{BLO}\left(\mathbb{R}^{n}\right)$, then we define $\|a\|_{\mathrm{BLO}\left(\mathbb{R}^{n}\right)}=\sup _{Q}\left\{m_{Q}(a)-\inf _{x \in Q} a(x)\right\}$.

Obviously, $L^{\infty}\left(\mathbb{R}^{n}\right) \subset \operatorname{BLO}\left(\mathbb{R}^{n}\right) \subset \operatorname{BMO}\left(\mathbb{R}^{n}\right)$, and if $a \in \operatorname{BLO}\left(\mathbb{R}^{n}\right)$, then

$$
\|a\|_{\mathrm{BMO}\left(\mathbb{R}^{n}\right)} \leq 2\|a\|_{\mathrm{BLO}\left(\mathbb{R}^{n}\right)} .
$$

Now let us formulate our main result as follows.

TheOREM 1. Suppose that $\Omega$ is homogeneous of degree zero on $\mathbb{R}^{n}$ and belongs to $B_{q}^{0,0}\left(S^{n-1}\right)$ for some $q>1$ with mean value zero on $S^{n-1}, a \in$ $\mathrm{BMO}\left(\mathbb{R}^{n}\right)$, and $P(x, y)$ is a real-valued polynomial. If $a \in \mathrm{BLO}\left(\mathbb{R}^{n}\right)$ and $a$ is subharmonic, then for $1<p<\infty$,

$$
\left\|T_{a, k} f\right\|_{p} \leq C\|a\|_{\mathrm{BLO}\left(\mathbb{R}^{n}\right)}^{k}\|f\|_{p},
$$

where $C$ is independent of the coefficients of $P(x, y)$.

REMARK 1. A typical example of a BMO function $a$ satisfying the restrictive conditions of Theorem 1 is $\log |x|$.

1.2. Commutators generated by a Lipschitz function. Let $\dot{\Lambda}_{\beta}\left(\mathbb{R}^{n}\right)$ denote the Lipschitz space defined by

$$
\dot{\Lambda}_{\beta}\left(\mathbb{R}^{n}\right)=\left\{f:\|f\|_{\dot{\Lambda}_{\beta}}=\sup _{x, h \in \mathbb{R}^{n} ; h \neq 0} \frac{\left|\Delta_{h}^{[\beta]+1} f(x)\right|}{|h|^{\beta}}<\infty\right\},
$$

where $\Delta_{h}^{1} f(x)=f(x+h)-f(x), \Delta_{h}^{k+1} f(x)=\Delta_{h}^{1}\left(\Delta_{h}^{k-1} f\right)(x)$. It is easy to see that if $0<\beta<1$ and $f \in \dot{\Lambda}_{\beta}$, then

$$
|f(x)-f(y)| \leq|x-y|^{\beta}\|f\|_{\dot{\Lambda}_{\beta}}, \quad \forall x, y \in \mathbb{R}^{n} .
$$

For $a \in \dot{\Lambda}_{\beta}\left(\mathbb{R}^{n}\right)$ and $k \in \mathbb{N}$, M. Paluszyński [19] considered the following commutators related to singular integrals:

$$
\bar{T}_{a, k} f(x)=\text { p.v. } \int_{\mathbb{R}^{n}} \frac{\Omega(x-y)}{|x-y|^{n}}(a(x)-a(y))^{k} f(y) d y,
$$

and proved that if $\Omega \in \operatorname{Lip}_{1}\left(S^{n-1}\right)$, then $\bar{T}_{a, k}$ is bounded from $L^{p}\left(\mathbb{R}^{n}\right)$ to $L^{r}\left(\mathbb{R}^{n}\right)$, where $1 / r=1 / p-\beta / n$ with $0<\beta<1$.

This result indicates that $\bar{T}_{a, k}$ enjoys the same mapping properties on the Lebesgue spaces as the fractional integral operators $\bar{T}_{\Omega, \beta}$ defined by (see e.g. $[17,18,8])$

$$
\bar{T}_{\Omega, \beta} f(x)=\text { p.v. } \int_{\mathbb{R}^{n}} \frac{\Omega(x-y)}{|x-y|^{n-\beta}} f(y) d y .
$$


This naturally leads to the question whether $T_{a, k}$ has the same boundedness properties as the fractional oscillatory integral operator. This problem will be resolved by our next Theorem 2 .

For fractional oscillatory singular integral operators with smooth kernel, Ricci and Stein [20] showed the following result.

TheOREm A. For each $d \geq 2$, there exists an $a_{d}>0$ such that whenever (i) $P(x, y)$ is a real polynomial of total degree $\leq d$, which is nontrivial in the sense that it cannot be written as $P_{0}(x)+P_{1}(y)$, and (ii) $K(x, y)$ is a function which satisfies $|K(x, y)| \leq C|x-y|^{-n+\beta},|\nabla K(x, y)| \leq C|x-y|^{-n+\beta-1}$, then the operator $T_{\beta}$ defined by

$$
T_{\beta} f(x)=\int_{\mathbb{R}^{n}} e^{i P(x, y)} K(x, y) f(y) d y
$$

is bounded on $L^{p}\left(\mathbb{R}^{n}\right)$, where $0<\beta<a_{d}(1 / 2-|1 / p-1 / 2|)$, and the $L^{p}$ norm of the operator does depend on the polynomial $P(x, y)$.

In 1996, Y. Ding [6] improved the above result as follows.

Theorem B. Suppose that $\Omega$ is homogeneous of degree zero on $\mathbb{R}^{n}$ and belongs to $L^{q}\left(S^{n-1}\right)$, and $P(x, y)=\sum_{|\xi| \leq r,|\eta| \leq l} c_{\xi \eta} x^{\xi} y^{\eta}$ is a nontrivial polynomial on $\mathbb{R}^{n} \times \mathbb{R}^{n}$. Consider the fractional oscillatory singular integral operator

$$
T_{\Omega, \beta} f(x)=\int_{\mathbb{R}^{n}} e^{i P(x, y)} \frac{\Omega(x-y)}{|x-y|^{n-\beta}} f(y) d y .
$$

(i) If $0<\beta<\min \{(l+r) / 2 r,(l+r) / 2 l\}$ and $q>1 /(1-\beta)$, then $T_{\Omega, \beta}$ is bounded on $L^{2}\left(\mathbb{R}^{n}\right)$.

(ii) If $1<p<\infty(p \neq 2), 0<\beta<\min \{(l+r) / 2 r,(l+r) / 2 l\}\{1 / 2-$ $|1 / p-1 / 2|\}$ and $q>1 /(1-\beta)$, then $T_{\Omega, \beta}$ is bounded on $L^{p}\left(\mathbb{R}^{n}\right)$. Here the $L^{p}$ norm of $T_{\Omega, \beta}$ depends on the value of $\sum_{|\xi|=r,|\eta|=l}\left|c_{\xi \eta}\right|$, but not on the other coefficients of $P(x, y)$.

In this paper, we will establish the following theorem.

TheOREm 2. Suppose that $\Omega$ is homogeneous of degree zero on $\mathbb{R}^{n}$ and belongs to $L^{q}\left(S^{n-1}\right)$ with $q>1, a \in \dot{\Lambda}_{\beta}\left(\mathbb{R}^{n}\right), 0<\beta<1$, and $P(x, y)=$ $\sum_{|\xi| \leq r,|\eta| \leq l} c_{\xi \eta} x^{\xi} y^{\eta}$ is a nontrivial polynomial on $\mathbb{R}^{n} \times \mathbb{R}^{n}$, that is, it cannot be written as $P_{0}(x)+P_{1}(y)$.

(i) If $0<\beta<\min \{(l+r) / 2 r k,(l+r) / 2 l k\}$ and $q>1 /(1-\beta)$, then

$$
\left\|T_{a, k} f\right\|_{2} \leq C(n, k, A, \operatorname{deg} P)\|a\|_{\dot{\Lambda}_{\beta}}^{k}\|f\|_{2} .
$$

(ii) If $1<p<\infty \quad(p \neq 2), 0<\beta<\min \{(l+r) / 2 r k,(l+r) / 2 l k\}\{1 / 2-$ $|1 / p-1 / 2|\}$ and $q>1 /(1-\beta)$, then

$$
\left\|T_{a, k} f\right\|_{p} \leq C(n, k, A, \operatorname{deg} P)\|a\|_{\dot{\Lambda}_{\beta}}^{k}\|f\|_{p} .
$$


Here $A=\left(\sum_{|\xi|=r,|\eta|=l}\left|c_{\xi \eta}\right|\right)^{1 /(r+l)}$ and $\operatorname{deg} P$ denotes the total degree of $P(x, y)$.

REMARK 2. We remark that the bound of the fractional oscillatory integral operator in Theorem A does depend on the coefficients of $P(x, y)$, but the bound of $T_{a, k}$ in our theorem, as those of $T_{\Omega, \beta}$ in Theorem B, depend only on the value of $\sum_{|\xi|=r,|\eta|=l}\left|c_{\xi \eta}\right|$, but not on the other coefficients of $P(x, y)$.

This paper is organized as follows. In Section 2, we will give some preliminary lemmas. Next we will prove Theorem 1 in Section 3. Finally, the proof of Theorem 2 will be given in Section 4. Some ideas in the proofs of our theorems are taken from $[20,15,6]$. Throughout the rest of this paper, $C$ always denotes positive constants that are independent of the essential variables but whose value may vary at each occurrence.

2. Some lemmas. Let us first give some lemmas, which are crucial in the proof of our main results.

Lemma 1 (see [14]). Let $\Omega, a, k$ be as in Theorem 1 . Then for $1<p$ $<\infty$, the maximal operator $M_{a, k}^{\Omega}$ defined by

$$
M_{a, k}^{\Omega} f(x)=\sup _{r>0} \frac{1}{r^{n}} \int_{|x-y|<r}|a(x)-a(y)|^{k}|\Omega(x-y) f(y)| d y
$$

satisfies

$$
\left\|M_{a, k}^{\Omega} f\right\|_{p} \leq C\|a\|_{\mathrm{BLO}\left(\mathbb{R}^{n}\right)}^{k}\|f\|_{p}
$$

Lemma 2 (see [14]). Let $a, k$ be as in Theorem $1, \Omega_{0}$ be homogeneous of degree zero on $\mathbb{R}^{n}$, and $1<p<\infty$. For $\lambda>1$, if $\Omega_{0} \in L^{\lambda}\left(S^{n-1}\right)$, then the operator

$$
M_{a, k}^{\Omega_{0}} f(x)=\sup _{r>0} \frac{1}{r^{n}} \int_{|x-y|<r}|a(x)-a(y)|^{k}|\Omega(x-y) f(y)| d y
$$

satisfies

$$
\left\|M_{a, k}^{\Omega_{0}} f\right\|_{p} \leq C\|a\|_{\mathrm{BLO}\left(\mathbb{R}^{n}\right)}^{k}\left\|\Omega_{0}\right\|_{L^{\lambda}\left(S^{n-1}\right)}\|f\|_{p}
$$

Lemma 3 (see [14]). Let $\Omega, a, k$ be as in Theorem 1 . Then the commutator of the singular operator $\bar{T}_{a, k}$ defined by

$$
\bar{T}_{a, k} f(x)=\text { p.v. } \int_{\mathbb{R}^{n}}(a(x)-a(y))^{k} \frac{\Omega(x-y)}{|x-y|^{n}} f(y) d y
$$

is bounded on $L^{p}\left(\mathbb{R}^{n}\right)$, for $1<p<\infty$, with norm bounded by $C\|a\|_{\mathrm{BLO}\left(\mathbb{R}^{n}\right)}^{k}$.

Lemma 4. Let $\Omega, a, k$ be as in Theorem $1, \varepsilon>0$, and $1<p<\infty$. Suppose that $P(x, y)$ is any real-valued polynomial on $\mathbb{R}^{n} \times \mathbb{R}^{n}$. If $T_{a, k}$ is 
bounded on $L^{p}\left(\mathbb{R}^{n}\right)$ with norm bounded by $C\|a\|_{\mathrm{BLO}\left(\mathbb{R}^{n}\right)}^{k}$, then its truncation defined by

$$
T_{\varepsilon, a, k} f(x)=\text { p.v. } \int_{|x-y|<\varepsilon} e^{i P(x, y)}(a(x)-a(y))^{k} \frac{\Omega(x-y)}{|x-y|^{n}} f(y) d y
$$

is also bounded on $L^{p}\left(\mathbb{R}^{n}\right)$ with norm bounded by $C\|a\|_{\mathrm{BLO}\left(\mathbb{R}^{n}\right)}^{k}$.

Proof. Decompose $\mathbb{R}^{n}$ as $\mathbb{R}^{n}=\bigcup_{d} I_{d}$, where each $I_{d}$ is a cube having side length $\varepsilon / 8 n$ and the cubes $I_{d}$ have disjoint interiors. Set $f_{d}=f \chi_{I_{d}}$. Since the support of $T_{\varepsilon, a, k} f_{d}$ is contained in a fixed multiple of $I_{d}$, the supports of the various terms $T_{\varepsilon, a, k} f_{d}$ have bounded overlaps and so we have

$$
\left\|T_{\varepsilon ; a, k} f\right\|_{p}^{p} \leq C \sum_{d}\left\|T_{\varepsilon, a, k} f_{d}\right\|_{p}^{p}
$$

Thus we may assume that $\operatorname{supp}(f) \subset I$ for some cube $I$ with side length $\varepsilon / 8 n$ and center at $x_{0}$. Write

$$
\int_{\mathbb{R}^{n}}\left|T_{\varepsilon ; a, k} f(x)\right|^{p} d x=\left(\int_{\left|x-x_{0}\right| \leq \varepsilon / 4 n}+\int_{\varepsilon / 4 n<\left|x-x_{0}\right| \leq 3 \varepsilon}+\int_{3 \varepsilon<\left|x-x_{0}\right|}\right)\left|T_{\varepsilon ; a, k} f(x)\right|^{p} d x .
$$

Since $\left|x-x_{0}\right|<\varepsilon / 4 n$ and $\left|y-x_{0}\right| \leq \varepsilon / 8 n$ imply $\left|x-x_{0}\right| \leq \varepsilon$, we have $T_{\varepsilon ; a, k} f(x)=T_{a, k} f(x)$. Thus, for the first term, by the $L^{p}$-boundedness of $T_{a, k}$, the desired estimate holds. When $\varepsilon / 4 n<\left|x-x_{0}\right| \leq 3 \varepsilon$, since $\left|y-x_{0}\right| \leq$ $\varepsilon / 8 n$, we have $c_{0} \varepsilon \leq|x-y| \leq c_{1} \varepsilon$ for some constants $c_{0}$ and $c_{1}$. Therefore

$$
\left|T_{\varepsilon ; a, k} f(x)\right| \leq \int_{c_{0} \varepsilon \leq|x-y| \leq c_{1} \varepsilon}|a(x)-a(y)|^{k} \frac{|\Omega(x-y)|}{|x-y|^{n}}|f(y)| d y \leq C M_{a, k}^{\Omega} f(x) .
$$

By Lemma 1, we get

$$
\left\|T_{\varepsilon ; a, k} f\right\|_{p} \leq C\left\|M_{a, k}^{\Omega} f\right\|_{p} \leq C\|a\|_{\mathrm{BLO}\left(\mathbb{R}^{n}\right)}^{k}\|f\|_{p},
$$

which is the estimate for the second term. When $3 \varepsilon<\left|x-x_{0}\right|$, we get $T_{\varepsilon ; a, k} f(x)=0$ and complete the proof of Lemma 4 .

Lemma 5 (see [19]). Let $0<\beta<1,1 \leq t<\infty$. We have

$$
\|f\|_{\dot{\Lambda}_{\beta}} \approx \sup _{Q} \frac{1}{|Q|^{\beta / n}}\left(\frac{1}{Q} \int_{Q}\left|f(x)-m_{Q}(f)\right|^{t} d x\right)^{1 / t},
$$

where $m_{Q}(f)=(1 /|Q|) \int_{Q} f(x) d x$. For $q=\infty$, the formula should be interpreted appropriately.

Lemma 6 (see [19]). Let $Q^{*} \subset Q, g \in \dot{\Lambda}_{\beta}(0<\beta<1)$. Then

$$
\left|m_{Q^{*}}(g)-m_{Q}(g)\right| \leq C|Q|^{\beta / n}\|g\|_{\dot{\Lambda}_{\beta}} .
$$

3. Proof of Theorem 1. The argument is by double induction on the degrees in $x$ and $y$ of the polynomial $P(x, y)$ as follows. If $P(x, y)$ is 
trivial, it is obvious that the assertion follows from Lemma 3 . For the general polynomial $P(x, y)$, let $r$ and $l$ be two strictly positive integers and write

$$
P(x, y)=\sum_{|\xi| \leq r,|\eta| \leq l} c_{\xi \eta} x^{\xi} y^{\eta} .
$$

By dilation invariance, we may assume that $\sum_{|\xi|=r,|\eta|=l}\left|c_{\xi \eta}\right|=1$. Now we assume the theorem is known for all polynomials which are sums of monomials of degree less than $r$ in $x$ times polynomials of any degree in $y$, and of monomials which are of degree $r$ in $x$ times monomials which are of degree less than $l$ in $y$. Rewrite

$$
P(x, y)=\sum_{|\xi|=r,|\eta|=l} c_{\xi \eta} x^{\xi} y^{\eta}+P_{0}(x, y),
$$

where $P_{0}(x, y)$ satisfies the inductive hypothesis. Decompose $T_{a, k}$ as

$$
\begin{aligned}
T_{a, k} f(x)= & \int_{|x-y|<1} e^{i P(x, y)} \frac{\Omega(x-y)}{|x-y|^{n}}(a(x)-a(y))^{k} f(y) d y \\
& +\int_{|x-y| \geq 1} e^{i P(x, y)} \frac{\Omega(x-y)}{|x-y|^{n}}(a(x)-a(y))^{k} f(y) d y \\
:= & T_{a, k}^{0} f(x)+T_{a, k}^{\infty} f(x) .
\end{aligned}
$$

It suffices to show that the estimate of the theorem holds for $T_{a, k}^{0}$ and $T_{a, k}^{\infty}$. Write

$$
\begin{aligned}
T_{a, k}^{0} f(x)= & \int_{|x-y|<1} e^{i P_{0}(x, y)} \frac{\Omega(x-y)}{|x-y|^{n}}(a(x)-a(y))^{k} f(y) d y \\
& +\int_{|x-y|<1}\left(e^{i P(x, y)}-e^{i P_{0}(x, y)}\right) \frac{\Omega(x-y)}{|x-y|^{n}}(a(x)-a(y))^{k} f(y) d y \\
:= & T_{a, k}^{0,1} f(x)+T_{a, k}^{0,2} f(x) .
\end{aligned}
$$

By the inductive hypothesis and Lemma 4 , we get

$$
\left\|T_{a, k}^{0,1} f\right\|_{p} \leq C\|a\|_{\mathrm{BLO}\left(\mathbb{R}^{n}\right)}^{k}\|f\|_{p} .
$$

On the other hand, note that if $|x|<1$ and $|x-y|<1$, then by (5),

$$
\left|e^{i P(x, y)}-e^{i P_{0}(x, y)}\right| \leq C \sum_{|\xi|=r,|\eta|=l}\left|c_{\xi \eta}\right||x-y|=C|x-y| .
$$

Set $f_{0}(y)=f(y) \chi_{\{|y| \leq 2\}}(y)$. Then $T_{a, k}^{0,2} f(x)=T_{a, k}^{0,2} f_{0}(x)$ if $|x|<1$. Thus

$$
\left|T_{a, k}^{0,2} f(x)\right| \leq C \int_{|x-y|<1} \frac{|\Omega(x-y)|}{|x-y|^{n-1}}|a(x)-a(y)|^{k}\left|f_{0}(y)\right| d y \leq C M_{a, k}^{\Omega} f(x) .
$$


It follows from Lemma 1 that

$$
\int_{|x|<1}\left|T_{a, k}^{0,2} f(x)\right|^{p} d x \leq C\|a\|_{\mathrm{BLO}\left(\mathbb{R}^{n}\right)}^{p k} \int_{|y|<2}|f(y)|^{p} d y .
$$

Using the same argument as in [20, p. 189], we obtain

$$
\int_{|x-t|<1}\left|T_{a, k}^{0,2} f(x)\right|^{p} d x \leq C\|a\|_{\mathrm{BLO}\left(\mathbb{R}^{n}\right)}^{p k} \int_{|y-t|<2}|f(y)|^{p} d y .
$$

Integrating the above inequality with respect to $t$, we have

$$
\left\|T_{a, k}^{0,2} f\right\|_{p} \leq C\|a\|_{\mathrm{BLO}\left(\mathbb{R}^{n}\right)}^{k}\|f\|_{p} .
$$

Putting (6) and (7) together shows that

$$
\left\|T_{a, k}^{0} f\right\|_{p} \leq C\|a\|_{\mathrm{BLO}\left(\mathbb{R}^{n}\right)}^{k}\|f\|_{p} .
$$

Next we turn to $T_{a, k}^{\infty}$. By the block decomposition of $\Omega$,

$$
\Omega\left(x^{\prime}\right)=\sum_{s} C_{s} b_{s}\left(x^{\prime}\right)
$$

we have

$$
\begin{aligned}
T_{a, k}^{\infty} f(x) & =\sum_{s} C_{s} \sum_{j=0}^{\infty} \int_{2^{j} \leq|x-y|<2^{j+1}} e^{i P(x, y)} \frac{b_{s}(x-y)}{|x-y|^{n}}(a(x)-a(y))^{k} f(y) d y \\
& :=\sum_{s} C_{s} \sum_{j=0}^{\infty} T_{a, k}^{j, s} f(x) .
\end{aligned}
$$

So

$$
\left\|T_{a, k}^{\infty} f\right\|_{p} \leq \sum_{s}\left|C_{s}\right| \sum_{j=0}^{\infty}\left\|T_{a, k}^{j, s} f\right\|_{p}
$$

By Lemma 2, for any $\lambda>1$ we have

$$
\left\|T_{a, k}^{j, s} f\right\|_{p} \leq C\left\|M_{a, k}^{b_{s}} f\right\|_{p} \leq C\left\|b_{s}\right\|_{L^{\lambda}\left(S^{n-1}\right)}\|a\|_{\mathrm{BLO}\left(\mathbb{R}^{n}\right)}^{k}\|f\|_{p} .
$$

Below, we shall give a more refined estimate on $\left\|T_{b, k}^{j, s} f\right\|_{2}$. Precisely, we shall show that for any $\lambda>1$,

$$
\left\|T_{a, k}^{j, s} f\right\|_{2} \leq C A^{\delta} 2^{-\delta j}\left\|b_{s}\right\|_{L^{\lambda}\left(S^{n-1}\right)}\|a\|_{\mathrm{BLO}\left(\mathbb{R}^{n}\right)}^{k}\|f\|_{2}
$$

uniformly for $\delta \in(0,1]$ such that $\delta<\min \left\{r / 2 l, r /(r+l) \lambda^{\prime}\right\}$, where $C, A$ depend only on $n$ and, in the latter case, $\operatorname{deg} P$.

By the dilation invariance again, we turn to the operators $\widetilde{T}^{j, s}$ and $\widetilde{T}_{a, k}^{j, s}$ defined by

$$
\widetilde{T}^{j, s} f(x)=\int_{1 \leq|x-y|<2} e^{i P\left(2^{j} x, 2^{j} y\right)} \frac{b_{s}(x-y)}{|x-y|^{n}} f(y) d y
$$


and

$$
\widetilde{T}_{a, k}^{j, s} f(x)=\int_{1 \leq|x-y|<2} e^{i P\left(2^{j} x, 2^{j} y\right)}(a(x)-a(y))^{k} \frac{b_{s}(x-y)}{|x-y|^{n}} f(y) d y .
$$

To prove (11), it suffices to prove that

$$
\left\|\widetilde{T}_{a, k}^{j, s} f\right\|_{2} \leq C A^{\delta} 2^{-\delta j}\left\|b_{s}\right\|_{L^{\lambda}\left(S^{n-1}\right)}\|a\|_{\mathrm{BLO}\left(\mathbb{R}^{n}\right)}^{k}\|f\|_{2},
$$

where $\delta, C$ and $A$ are as in (11).

As in the proof of Lemma 4 , split $\mathbb{R}^{n}$ into cubes $I_{d}$ having side length 1 and disjoint interiors. Set $f_{d}=f \chi_{I_{d}}$. Similarly to the proof of Lemma 4, we have

$$
\left\|\widetilde{T}_{a, k}^{j, s} f\right\|_{2}^{2} \leq C \sum_{d}\left\|\widetilde{T}_{a, k}^{j, s} f_{d}\right\|_{2}^{2} .
$$

Thus we may assume that $\operatorname{supp}(f) \subset I$ for a cube $I$ with side length 1 . Choose $\varphi \in C_{0}^{\infty}\left(\mathbb{R}^{n}\right), 0 \leq \varphi \leq 1, \varphi$ is identically one on $50 n I$ and vanishes outside 100nI. Write $\bar{I}=100 n I$ and $\widetilde{a}(x)=\left(a(x)-m_{\bar{I}}(a)\right) \varphi(x)$, where $m_{\bar{I}}(a)$ is the mean value of $a$ on $\bar{I}$. When $y \in I$ and $x$ is in the support of $\widetilde{T}_{a, k}^{j, s} f$, we have

$$
(a(x)-a(y))^{k}=\sum_{m=0}^{k}(-1)^{k-m} C_{k}^{m} \widetilde{a}^{m}(x) \widetilde{a}^{k-m}(y)
$$

and

$$
\widetilde{T}_{a, k}^{j, s} f(x)=\sum_{m=0}^{k}(-1)^{k-m} C_{k}^{m} \widetilde{a}^{m}(x) \widetilde{T}^{j, s}\left(\widetilde{a}^{k-m} f\right)(x) .
$$

For each fixed integer $m, 0 \leq m \leq k$, notice that $\operatorname{supp}\left(\widetilde{T}^{j, s}\left(\widetilde{a}^{k-m} f\right)\right) \subset 20 n I$. We first claim that for any $\lambda>1$,

$$
\left\|\widetilde{T}^{j, s} f\right\|_{2} \leq C A^{\delta} 2^{-\delta j}\left\|b_{s}\right\|_{L^{\lambda}\left(S^{n-1}\right)}\|f\|_{2},
$$

where $C, A$ and $\delta$ are as in (11).

In fact, consider the operator

$$
T^{j, s} f(x)=\int_{2^{j} \leq|x-y|<2^{j+1}} e^{i P(x, y)} \frac{b_{s}(x-y)}{|x-y|^{n}} f(y) d y .
$$

By Propositions 2 and 3 in [13], carefully inspecting the proof in [15, pp. 209$213]$ and making a minor modification, we easily obtain

$$
\left\|T^{j, s} f\right\|_{2} \leq C A^{\delta} 2^{-\delta j}\left\|b_{s}\right\|_{L^{\lambda}\left(S^{n-1}\right)}\|f\|_{2},
$$

where $C, A$ and $\delta$ are as in (13). So (13) follows by dilation invariance.

Now recalling that $a(x)$ is subharmonic, we have

$$
\|\widetilde{a}\|_{\infty}=\sup _{x \in \bar{I}}\left|a(x)-m_{\bar{I}}(a)\right|=\sup _{x \in \bar{I}}\left\{m_{\bar{I}}(a)-a(x)\right\} \leq m_{\bar{I}}-\inf _{x \in \bar{I}} a(x) \leq\|a\|_{\operatorname{BLO}\left(\mathbb{R}^{\mathrm{n}}\right)} .
$$


Therefore, by (13) we get

$$
\begin{aligned}
\left\|\widetilde{a}^{m} \widetilde{T}^{j, s}\left(\widetilde{a}^{k-m} f\right)\right\|_{2} & \leq\left\|\widetilde{a}^{m}\right\|_{\infty}\left\|\widetilde{T}^{j, s}\left(\left|\widetilde{a}^{k-m} f\right|\right)\right\|_{2} \\
& \leq C A^{\delta} 2^{-\delta j}\|a\|_{\mathrm{BLO}\left(\mathbb{R}^{n}\right)}^{m}\left\|b_{s}\right\|_{L^{\lambda}\left(S^{n-1}\right)}\left\|\widetilde{a}^{k-m} f\right\|_{2} \\
& \leq C A^{\delta} 2^{-\delta j}\|a\|_{\mathrm{BLO}\left(\mathbb{R}^{n}\right)}^{m}\left\|b_{s}\right\|_{L^{\lambda}\left(S^{n-1}\right)}\left\|\widetilde{a}^{k-m}\right\|_{\infty}\|f\|_{2} \\
& \leq C A^{\delta} 2^{-\delta j}\|a\|_{\mathrm{BLO}\left(\mathbb{R}^{n}\right)}^{k}\left\|b_{s}\right\|_{L^{\lambda}\left(S^{n-1}\right)}\|f\|_{2} .
\end{aligned}
$$

Summing over $m$, we obtain (12) and complete the proof of (11).

Therefore, interpolation between (10) and (11) shows that

$$
\left\|T_{a, k}^{j, s} f\right\|_{p} \leq C A^{\theta \delta} 2^{-\theta \delta j}\left\|b_{s}\right\|_{L^{\lambda}\left(S^{n-1}\right)}\|a\|_{\mathrm{BLO}\left(\mathbb{R}^{n}\right)}^{k}\|f\|_{p}
$$

for $1<p<\infty$, where $0<\theta \leq 1$.

Now we return to the estimate of $\left\|T_{a, k}^{\infty} f\right\|_{p}$. Recall that for each $b_{s}$, $\operatorname{supp}\left(b_{s}\right) \subset Q_{s}$ and $\left\|b_{s}\right\|_{L^{q}\left(S^{n-1}\right)} \leq\left|Q_{s}\right|^{1 / q-1}$. If $\left|Q_{s}\right| \geq e^{q /(1-q)}$, we take $\lambda=q$. Then it follows from (14) that

$\left\|T_{a, k}^{j, s} f\right\|_{p} \leq C A^{\delta} 2^{-\theta \delta j}\|a\|_{\mathrm{BLO}\left(\mathbb{R}^{n}\right)}^{k}\left\|b_{s}\right\|_{L^{q}\left(S^{n-1}\right)}\|f\|_{p} \leq C 2^{-\theta \delta j}\|a\|_{\mathrm{BLO}\left(\mathbb{R}^{n}\right)}^{k}\|f\|_{p}$.

So

$$
\sum_{j \geq 0}\left\|T_{a, k}^{j, s} f\right\|_{p} \leq C \sum_{j \geq 0} 2^{-\theta \delta j}\|a\|_{\mathrm{BLO}\left(\mathbb{R}^{n}\right)}^{k}\|f\|_{p} \leq C\|a\|_{\mathrm{BLO}\left(\mathbb{R}^{n}\right)}^{k}\|f\|_{p} .
$$

If $\left|Q_{s}\right|<e^{q /(1-q)}$, we take $\lambda=\log \left|Q_{s}\right| /\left(1+\log \left|Q_{s}\right|\right)$ and choose $\delta=\sigma / \lambda^{\prime}<$ $\min \left\{r / 2 l, r /(r+l) \lambda^{\prime}\right\}$, where $\sigma$ is a positive constant depending only on $r$ and $l$. Then (14) leads again to

$$
\begin{aligned}
\left\|T_{a, k}^{j, s} f\right\|_{p} & \leq C A^{\sigma / \lambda^{\prime}} 2^{-j \theta \sigma / \lambda^{\prime}}\left\|b_{s}\right\|_{L^{\lambda}\left(S^{n-1}\right)}\|a\|_{\mathrm{BLO}\left(\mathbb{R}^{n}\right)}^{k}\|f\|_{p} \\
& \leq C 2^{j \theta \sigma / \log \left|Q_{s}\right|}\left|Q_{s}\right|^{1 / \lambda-1}\|a\|_{\mathrm{BLO}\left(\mathbb{R}^{n}\right)}^{k}\|f\|_{p} \\
& \leq C 2^{j \theta \sigma / \log \left|Q_{s}\right|}\|a\|_{\mathrm{BLO}\left(\mathbb{R}^{n}\right)}^{k}\|f\|_{p} .
\end{aligned}
$$

So

$$
\begin{aligned}
\sum_{j \geq 0}\left\|T_{a, k}^{j, s} f\right\|_{p} & \leq C\|a\|_{\mathrm{BLO}\left(\mathbb{R}^{n}\right)}^{k} \sum_{j \geq 0} 2^{j \theta \sigma / \log \left|Q_{s}\right|}\|f\|_{p} \\
& \leq C\|a\|_{\mathrm{BLO}\left(\mathbb{R}^{n}\right)}^{k} \log \frac{1}{\left|Q_{s}\right|}\|f\|_{p} .
\end{aligned}
$$

Therefore, by (9) we obtain

$$
\begin{aligned}
\left\|T_{a, k}^{\infty} f\right\|_{p} & \leq C \sum_{s}\left|C_{s}\right|\left(1+\log ^{+} \frac{1}{\left|Q_{s}\right|}\right)\|a\|_{\mathrm{BLO}\left(\mathbb{R}^{n}\right)}^{k}\|f\|_{p} \\
& \leq C\|a\|_{\mathrm{BLO}\left(\mathbb{R}^{n}\right)}^{k}\|f\|_{p} .
\end{aligned}
$$

This completes the proof of Theorem 1. 
4. Proof of Theorem 2. Similarly to the proof of Theorem 1, we write

$$
\begin{aligned}
T_{a, k} f(x)= & \int_{|x-y|<1} e^{i P(x, y)} \frac{\Omega(x-y)}{|x-y|^{n}}(a(x)-a(y))^{k} f(y) d y \\
& +\int_{|x-y| \geq 1} e^{i P(x, y)} \frac{\Omega(x-y)}{|x-y|^{n}}(a(x)-a(y))^{k} f(y) d y \\
= & : T_{a, k}^{0} f(x)+T_{a, k}^{\infty} f(x) .
\end{aligned}
$$

We will establish the $L^{p}$-boundedness of $T_{a, k}$ in the following two cases:

CASE 1. $\sum_{|\xi|=r,|\eta|=l}\left|c_{\xi \eta}\right|=1$. First, we estimate $\left\|T_{a, k}^{0} f\right\|_{p}, 1<p<\infty$. By (4), we have

$$
\begin{aligned}
\left|T_{a, k}^{0} f(x)\right| & \leq \int_{|x-y|<1} \frac{|\Omega(x-y)|}{|x-y|^{n}}|a(x)-a(y)|^{k}|f(y)| d y \\
& =\sum_{j=0}^{\infty} \int_{2^{-j-1} \leq|x-y|<2^{-j}} \frac{|\Omega(x-y)|}{|x-y|^{n}}|a(x)-a(y)|^{k}|f(y)| d y \\
& \leq C \sum_{j=0}^{\infty} 2^{-j k \beta}\|a\|_{\dot{\Lambda}_{\beta}}^{k} \int_{2^{-j-1} \leq|x-y|<2^{-j}} \frac{|\Omega(x-y)|}{|x-y|^{n}}|f(y)| d y \\
& \leq C\|a\|_{\dot{\Lambda}_{\beta}}^{k} \sum_{j=0}^{\infty} 2^{-j k \beta} 2^{j n} \int_{|x-y|<2^{-j}}|\Omega(x-y)||f(y)| d y \\
& \leq C\|a\|_{\dot{\Lambda}_{\beta}}^{k} \sum_{j=0}^{\infty} 2^{-j k \beta} M_{\Omega} f(x) \leq C\|a\|_{\dot{\Lambda}_{\beta}}^{k} M_{\Omega} f(x),
\end{aligned}
$$

where $M_{\Omega}$ is the maximal operator with rough kernel defined by

$$
M_{\Omega} f(x)=\sup _{R>0} \frac{1}{R^{n}} \int_{|x-y|<R}|\Omega(x-y)||f(y)| d y .
$$

Since $\Omega \in L^{q}\left(S^{n-1}\right)(q>1)$, from [2] we get

$$
\left\|M_{\Omega} f\right\|_{p} \leq C\|f\|_{p}, \quad 1<p<\infty .
$$

Thus

$$
\left\|T_{a, k}^{0} f\right\|_{p} \leq C\|a\|_{\dot{\Lambda}_{\beta}}^{k}\left\|M_{\Omega} f\right\|_{p} \leq C\|a\|_{\dot{\Lambda}_{\beta}}^{k}\|f\|_{p},
$$

where $C$ is independent of the coefficients of $P(x, y)$. It remains to show that

$$
\left\|T_{a, k}^{\infty} f\right\|_{p} \leq C\|a\|_{\dot{\Lambda}_{\beta}}^{k}\|f\|_{p}
$$


Set

$$
T_{a, k}^{j} f(x)=\int_{2^{j} \leq|x-y|<2^{j+1}} e^{i P(x, y)} \frac{\Omega(x-y)}{|x-y|^{n}}(a(x)-a(y))^{k} f(y) d y .
$$

Then

$$
T_{a, k}^{\infty} f(x)=\sum_{j=0}^{\infty} T_{a, k}^{j} f(x) .
$$

So we only need to prove that there exists a constant $\theta>0$ such that for every $0 \leq j<\infty$,

$$
\left\|T_{a, k}^{j} f\right\|_{p} \leq C\|a\|_{\dot{A}_{\beta}}^{k} 2^{-j \theta}\|f\|_{p},
$$

where $C$ is independent of $f$ and $j$.

As in the proof of (11), we turn our attention to the operator

$$
\widetilde{T}_{a, k}^{j} f(x)=2^{j k \beta} \int_{1 \leq|x-y|<2} e^{i P\left(2^{j} x, 2^{j} y\right)} \frac{\Omega(x-y)}{|x-y|^{n}}(a(x)-a(y))^{k} f(y) d y .
$$

It is easy to observe that the proof of (19) can be reduced to showing that

$$
\left\|\widetilde{T}_{a, k}^{j} f\right\|_{p} \leq C\|a\|_{\dot{\Lambda}_{\beta}}^{k} 2^{-j \theta}\|f\|_{p} .
$$

As in the proof of Theorem 1 , we split $\mathbb{R}^{n}$ into cubes $Q_{d}$ having side length 1 and disjoint interiors. Set $f_{d}=f \chi_{Q_{d}}$. Similarly to the arguments in Lemma 4, we have

$$
\left\|\widetilde{T}_{a, k}^{j} f\right\|_{p}^{p} \leq C \sum_{d}\left\|\widetilde{T}_{a, k}^{j} f_{d}\right\|_{p}^{p} .
$$

Thus we may assume that $\operatorname{supp}(f) \subset Q$ for a cube $Q$ with side length 1 . Choose $\phi \in C_{0}^{\infty}\left(\mathbb{R}^{n}\right)$ such that $0 \leq \phi \leq 1, \phi$ is identically one on $50 n Q$ and vanishes outside $100 n Q$. Define $\bar{Q}=100 n Q$ and $\widetilde{a}(x)=\left(a(x)-m_{\bar{Q}}(a)\right) \phi(x)$, where $m_{\bar{Q}}(a)$ is the mean value of $a$ on $\bar{Q}$. It is easy to deduce from Lemmas $5-6$ that

$$
\|\widetilde{a}\|_{\infty} \leq C|\bar{Q}|^{\beta / n}\|a\|_{\dot{\Lambda}_{\beta}} \leq C\|a\|_{\dot{\Lambda}_{\beta}} .
$$

When $y \in Q$ and $x$ is in the support of $\widetilde{T}_{a, k}^{j} f$, we have

$$
(a(x)-a(y))^{k}=\sum_{m=0}^{k}(-1)^{k-m} C_{k}^{m} \widetilde{a}^{m}(x) \widetilde{a}^{k-m}(y)
$$

and

$$
\widetilde{T}_{a, k}^{j} f(x)=\sum_{m=0}^{k}(-1)^{k-m} C_{k}^{m} \widetilde{a}^{m}(x) \widetilde{T}^{j}\left(\widetilde{a}^{k-m} f\right)(x),
$$


where $\widetilde{T}^{j}$ is defined by

$$
\widetilde{T}^{j} h(x)=2^{j k \beta} \int_{1 \leq|x-y|<2} e^{i P\left(2^{j} x, 2^{j} y\right)} \frac{\Omega(x-y)}{|x-y|^{n}} h(y) d y .
$$

We claim that there exists a positive constant $\theta>0$ such that

$$
\left\|\widetilde{T}^{j} h\right\|_{p} \leq C 2^{-j \theta}\|h\|_{p}, \quad 1<p<\infty .
$$

In fact, we consider the following operator:

$$
T^{j} h(x)=2^{j k \beta} \int_{2^{j} \leq|x-y|<2^{j+1}} e^{i P(x, y)} \frac{\Omega(x-y)}{|x-y|^{n}} h(y) d y .
$$

Carefully inspecting the arguments of Ding in [6, pp. 73-79], under the assumptions of Theorem 2 we can deduce that there exists a positive constant $\delta=\delta(n, \operatorname{deg} P)$ such that

(i) $\beta-\delta-\delta l / r<0$ and $\delta<\min \left\{r / 2 k l, r / k q^{\prime}(r+l)\right\}$ and

$$
\left\|T^{j} h\right\|_{2} \leq C 2^{(\beta-\delta-\delta l / r) j}\|h\|_{2} ;
$$

(ii) $\beta-\delta \sigma-\delta \sigma l / r<0$ and $\delta<\min \left\{1 / 2 k, r / 2 l k, r / k q^{\prime} \sigma(r+l)\right\}$ and

$$
\left\|T^{j} h\right\|_{p} \leq C 2^{(\beta-\delta \sigma-\delta \sigma l / r) j}\|h\|_{p},
$$

where $1<p<\infty \quad(p \neq 2), \sigma=1 / 2-|1 / p-1 / 2|$. Here $C$ is independent of the coefficients of $P(x, y)$.

Therefore, by dilation invariance we obtain

$$
\left\|\widetilde{T}^{j} h\right\|_{p} \leq C 2^{-j \theta}\|h\|_{p}
$$

where $\theta=\delta+\delta l / r-\beta>0$ for $p=2$, and $\theta=\delta(1 / 2-|1 / p-1 / 2|)+\delta(1 / 2-$ $|1 / p-1 / 2|) l / r-\beta>0$ for $1<p<\infty \quad(p \neq 2)$. This proves $(22)$.

Now we estimate $\left\|\widetilde{T}_{a, k}^{j} f\right\|_{p}$. For each fixed integer $m, 0 \leq m \leq k$, noticing that $\operatorname{supp}\left(\widetilde{T}^{j}\left(\widetilde{a}^{k-m} f\right)\right) \subset 20 n Q$ and $\|\widetilde{a}\|_{\infty} \leq C\|a\|_{\dot{\Lambda}_{\beta}}$, by (22) we get

$$
\begin{aligned}
\left\|\widetilde{a}^{m} \widetilde{T}^{j}\left(\widetilde{a}^{k-m} f\right)\right\|_{p} & \leq\left\|\widetilde{a}^{m}\right\|_{\infty}\left\|\widetilde{T}^{j}\left(\widetilde{a}^{k-m} f\right)\right\|_{p} \leq C\|a\|_{\dot{\Lambda}_{\beta}}^{m} 2^{-j \theta}\left\|\widetilde{a}^{k-m} f\right\|_{p} \\
& \leq C\|a\|_{\dot{\Lambda}_{\beta}}^{m} 2^{-j \theta}\left\|\widetilde{a}^{k-m}\right\|_{\infty}\|f\|_{p} \leq C\|a\|_{\dot{\Lambda}_{\beta}}^{k} 2^{-j \theta}\|f\|_{p} .
\end{aligned}
$$

From (21), summing the above inequality over $m$, we obtain (20). This completes the proof of Theorem 2 in the case $\sum_{|\xi|=r,|\eta|=l}\left|c_{\xi \eta}\right|=1$.

CASE 2. $\sum_{|\xi|=r,|\eta|=l}\left|c_{\xi \eta}\right| \neq 1$. Letting $A$ be as in Theorem 2, we can write $P(x, y)$ as follows,

$$
P(x, y)=\sum_{|\xi|=r,|\eta|=l} \frac{c_{\xi \eta}}{A^{r+l}}(A x)^{\xi}(A y)^{\eta}+R_{0}\left(\frac{A x}{A}, \frac{A y}{A}\right)=: Q(A x, A y) .
$$


Then

$$
\begin{aligned}
T_{a, k} f(x) & =\int_{\mathbb{R}^{n}} e^{i Q(A x, A y)}(a(x)-a(y))^{k} \frac{\Omega(x-y)}{|x-y|^{n}} f(y) d y \\
& =\int_{\mathbb{R}^{n}} e^{i Q(A x, y)}\left(a\left(\frac{A x}{A}\right)-a\left(\frac{y}{A}\right)\right)^{k} \frac{\Omega(A x-y)}{|A x-y|^{n}} f\left(\frac{y}{A}\right) d y .
\end{aligned}
$$

Consequently,

$$
T_{a, k} f\left(\frac{x}{A}\right)=\int_{\mathbb{R}^{n}} e^{i Q(x, y)}\left(a\left(A^{-1} x\right)-a\left(A^{-1} y\right)\right)^{k} \frac{\Omega(x-y)}{|x-y|^{n}} f\left(\frac{y}{A}\right) d y .
$$

Since $\left\|a\left(A^{-1} \cdot\right)\right\|_{\dot{\Lambda}_{\beta}}=A^{-\beta}\|a\|_{\dot{\Lambda}_{\beta}}$, by the result proved in Case 1 , we obtain

$$
\left\|T_{a, k} f\right\|_{p} \leq C A^{-k \beta}\|a\|_{\dot{\Lambda}_{\beta}}^{k}\|f\|_{p}=C(n, k, A, \operatorname{deg} P)\|a\|_{\dot{\Lambda}_{\beta}}^{k}\|f\|_{p} .
$$

Theorem 2 is proved.

Acknowledgements. The author would like to thank the referee for his valuable comments and suggestions. The author is also grateful to Professor Shanzhen Lu for his encouragement and guidance.

\section{References}

[1] A. J. Al-Hasan and D. S. Fan, A singular integral operator related to block spaces, Hokkaido Math. J. 28 (1999), 285-299.

[2] A. P. Calderón and A. Zygmund, On singular integrals, Amer. J. Math. 78 (1956), 289-309.

[3] S. Chanillo and M. Christ, Weak $(1,1)$ bounds for oscillatory singular integrals, Duke Math. J. 55 (1987), 141-155.

[4] R. Coifman and R. Rochberg, Another characterization of BMO, Proc. Amer. Math. Soc. 79 (1980), 249-254.

[5] Y. Ding, Some problems on oscillatory singular integrals and fractional integrals with rough kernel, Ph.D. thesis, Beijing Normal Univ., 1995.

[6] - $L^{p}$-boundedness for fractional oscillatory integral operator with rough kernel, Approx. Theory Appl. 12 (1996), no. 2, 70-79.

[7] Y. Ding and S. Z. Lu, Weighted $L^{p}$-boundedness for higher order commutators of oscillatory singular integrals, Tôhoku Math. J. 48 (1996), 437-449.

[8] - - - Weighted norm inequalities for fractional integral operators with rough kernel, Canad. J. Math. 50 (1998), 29-39.

[9] G. Hu, Weighted norm inequalities for commutators of homogeneous singular integrals, Acta Math. Sinica (N.S.) 11 (1995), Special Issue, 77-88.

[10] Y. Jiang and S. Z. Lu, Oscillatory singular integrals with rough kernel, in: Harmonic Analysis in China, M. Cheng et al. (eds.), Kluwer, 1995, 135-145.

[11] M. Keitoku and S. Sato, Block spaces on the unit sphere in $\mathbb{R}^{n}$, Proc. Amer. Math. Soc. 119 (1993), 453-455.

[12] S. Z. Lu, M. Taibleson, and G. Weiss, Spaces Generated by Blocks, Beijing Normal Univ. Press, Beijing, 1989. 
[13] S. Z. $\mathrm{Lu}$ and $\mathrm{H} . \mathrm{Wu}$, Oscillatory singular integrals and commutators with rough kernels, Ann. Math. Québec 27 (2003), 47-66.

[14] - - - On the commutators for singular integrals related to block spaces, Nagoya Math. J. 173 (2004), 205-223; Corrections, 175 (2004), 223-224.

[15] S. Z. Lu and Y. Zhang, Criterion on $L^{p}$-boundedness for a class of oscillatory singular integrals with rough kernels, Rev. Mat. Iberoamericana 8 (1992), 201-219.

[16] B. Ma and $\mathrm{G}$. $\mathrm{Hu}, L^{2}\left(\mathbb{R}^{n}\right)$ boundedness for commutators of oscillatory singular integral operators, Approx. Theory Appl. 16 (2000), no. 2, 37-44.

[17] B. Muckenhoupt and R. L. Wheeden, Weighted norm inequalities for singular and fractional integrals, Trans. Amer. Math. Soc. 161 (1971), 249-258.

[18] - - - Weighted norm inequalities for fractional integrals, ibid. 192 (1974), 261-274.

[19] M. Paluszyński, Characterization of the Besov spaces via the commutator operator of Coifman, Rochberg and Weiss, Indiana Univ. Math. J. 44 (1995), 1-17.

[20] F. Ricci and E. M. Stein, Harmonic analysis on nilpotent groups and singular integrals, I. Oscillatory integrals, J. Funct. Anal. 73 (1987), 179-194.

[21] M. H. Taibleson and G. Weiss, Certain function spaces connected with almost everywhere convergence of Fourier series, in: Conf. on Harmonic Analysis in Honor of Antoni Zygmund (Chicago, 1981), Vol. I, Wadsworth, 1983, 95-113.

School of Mathematical Sciences

Xiamen University

Xiamen 361005

People's Republic of China

E-mail: huoxwu@xmu.edu.cn

Received August 14, 2003

Revised version October 1, 2004 